\title{
COASTAL CURRENTS MONITORING USING RADAR SATELLITES BASED ON WAVE TRACKING APPROACH
}

\author{
A. Abedini ${ }^{1}$, M. Aghamohamadnia, M. Sharifi, S. Farzaneh \\ Dept. of Surveying and Geomatics, Faculty of Engineering, University of Tehran, Tehran, Iran \\ (aabedeni, maghamohamadnia, sharifi, farzaneh) @ ut.ac.ir
}

Commission VIII/4

KEY WORDS: Coastal Currents, Monitoring, Jason1 Satellite, Wave Tracking, Radar Satellites

\begin{abstract}
:
Use of high resolution radar imagery for coastal currents monitoring has been known as an active topic in several research institutions. In this study, we demonstrate a new method for coastal currents monitoring based on current tracking by deformed wave reconstruction.

The magnitude and shape of the echoes (or waveforms) hold information regarding the surface characteristics that can be described analytically. Accordingly from this shape, several parameters can be construed, by comparing the real waveform with the theoretical curve. Safety in coastal zones containing coastal engineering for construction of ports, marine structures, beach erosion, natural hazards, and environmental problems are the issues related to hydrodynamic studies of currents, waves, and winds. Therefore, monitoring of coastal currents is very important . The ability of the internet to accompany our results to track past and near real-time movements of every coastal waters for monitoring is appropriate. Surface Current Mapping is utilized using an interface to radar altimetry data derived surface currents. Then Data access is available in a number of formats and protocols such as Google Earth KML and so on. In this study Jason1 satellite data has been used as input data for assessing the coastal environment.
\end{abstract}

\section{INTRODUCTION}

A ltimetry is basically a technique for measuring height. Satellite radar altimetry measures the time taken by a radar pulse to travel from the satellite antenna to the surface and back to the satellite receiver. Moreover, this measurement yields a wealth of other information that can be used for a wide range of applications ${ }^{[1]}$.

Many current studies are attempting to enhance the quality of altimetry data close to the coasts. New processing methods and applications can then be developed for littoral and shallowwater regions, some of the most fragile and important areas of the oceans.

There is a shortage of altimetry data near the coasts (or their inferior quality) which is caused due to several factors:

The technique itself, since the radar echoes reflected off water, and off a combination of water and land are not identical, and basically only the former undergo processing by the ground segments.

Other altimetry satellite measurements also suffer from the same problem, such as those from the radiometer (at a distance of about $50 \mathrm{~km}$ from the coast) ${ }^{[2]}$. But even this amount of data needs to be used in a way which is effective and efficient.

Since the satellite altimetry and radar observatory came to existence, various approaches have been offered to use their data in solving environmental problems and at most to construe and derive out valuable and profiting information to use in various areas of research.

An alternative method for calculating surface currents is through the exploitation of the Doppler shift in synthetic aperture radar data (Chapron, 2005) ${ }^{[3]}$. This method has promise in particular for coastal zones, although there are many technical challenges, including isolating the surface currents from signals such as wave orbital motion or tides.

Another method is a feature tracking algorithm to monitor ocean currents by using satellite sequential images of surface water (V.M.Krasnopolsky and et all, 1999) ${ }^{[4]}$.

One area of research is in waveform re-tracking reprocessing individual satellite track signals to recover the distorted waveform as it reaches land (Deng and Featherstone, 2006) ${ }^{[5]}$.

One of the new ways of monitoring the coastal areas with radar technology is using the HF radar monitoring system. HF radar tracks currents and eddies of coastal surface waters from just beyond the surf zone to $150 \mathrm{~km}$ offshore. The movement of these surface waters can be viewed in near real-time in a Google Maps interface or at web pages developed for specific users. If an oil spill occurs, these maps and products can be used to track where the oil is moving, even at night or in dense fog and extreme weather. Similar maps and websites allow city environmental managers to follow the trajectory of coastal discharges(like sewage spills or coastal runoff after heavy rains)so that only the affected beaches are targeted for water safety testing and subsequent closures when needed to protect our health.

In this paper unlike using pressure based or radar received wave power analyse which are examples of physical properties of water wave, the geometrical aspect of surface water wave is under concentration. So this can be described as a numerical and mathematical approach which applies on the oceanic water surface model taken from the altimetry data over a specific area [6].

\footnotetext{
${ }^{1}$ Corresponding author
} 
second momentum of area of the wave's three-dimensional surface peak. Based on the mathematical equations below Eq1 the surface relative extremes can be found.

\section{THE EXPLANATION OF METHODE AND ALGORITHMS}

As mentioned in the previous section, there are various approaches to achieve the velocity and direction of surface water waves in order to be used in the process of finding and extracting properties and features of ocean surface waters. In this paper concentration is on achieving the direction of surface currents in coastal areas, and unlike the most of other common works it is based on a geometrical method applied on the water surface digital elevation model.

Surface DEM can be obtained by exerting interpolation or Delaunay algorithms on a set of elevation points acquired from a surface ${ }^{[7][8][9][10]}$. This model has a variable extent depending on vastness of points taken through radar scan in a single epoch or several epochs got from techniques applied to discrete or gap data ${ }^{[11]}$. This model is a continuous surface of water surface shape at the time of scan and displays the topography of water which includes the wave peaks and lows.

There are 3 dimensional peaks and lows of small and large wave length resulted from tide or surface winds or any other external source of force. The surface winds produce waves with short wave lengths which have different shapes depending on the depth of water. So their shapes are different in the coastal or near coastal areas in comparison with far shore waters.

The waters in the coastal and near coastal zones are much likely to have an oriented wave shape and also with significant elevation difference between waves peaks and lows. In the presence of surface winds this shape deviates toward the wind direction.

It is possible to construe information of external forces from deformed shape of water wave. One of the information is direction of external force applied to water surface. The behavior analysis of this elevation model depends on the spatial resolution of data attained from radar altimetry satellite. Currently because of achieving high accuracy in satellite orbital coordinates (with basic measurement accuracy in the range $2 \mathrm{~cm}$ to $4 \mathrm{~cm})$ [12][13] it is possible to have accuracy a few centimetres over elevation coordinates which can help monitor even not very strong surface currents.
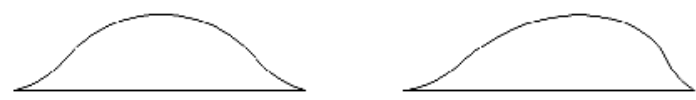

Figure 1. a) A typically normal wave peak shape (Right) b) A typically deformed wave peak shape by any external forces

In this paper it is assumed that the surface model of water is available in the coastal areas. This surface model has peaks and lows which can be described in detail. In the figl a wave peak is displayed in both normal and deformed by external forces shapes. Wave's peaks shapes in deep waters like oceans are mainly symmetric fig1-a but in low depth and coastal waters are deformed and have an attitude in line with the wind's direction fig1-b.

The method offered in this article takes profit of this behavior and exerts mathematical analysis on it in order to find out currents and consequently wind's direction. The analysis is related to the vector connecting points between the first and

$$
\left\{\mathrm{P}=(x, y, H) \text { is Fxtramim } \mid \frac{\partial H}{\partial x}=0, \frac{\partial H}{\partial y}=0\right\}
$$

By defining a local Cartesian coordinate system in the neighborhood of the maximum point it would be easier to perform computations on coordinates of points in a way which its $\mathrm{Z}$ axis approximately and without much need of precision be in line with gravity direction regardless of its direction and also with an arbitrary X-Y plane orientation.

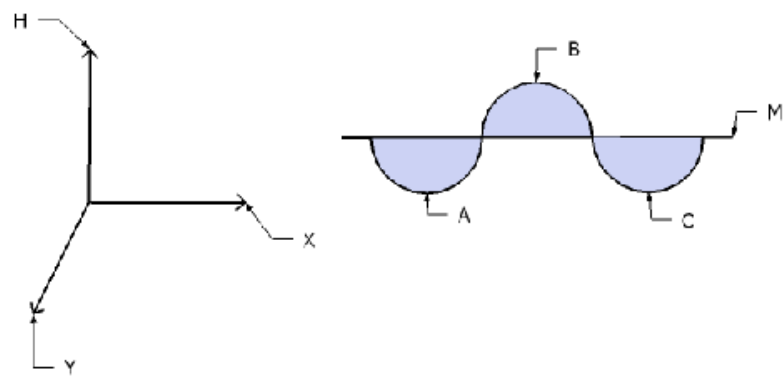

Figure 2. A wave peak and its neighbour low points in a 2-D view and the arbitrary local Cartesian coordinate system

Having extremes location and performing the algorithm below $\mathrm{Eq} 2$, it is possible to determine the maximum points limit range to be used in the analysis Fig3.

$H m=1 / 2\left(H b+1 / n\left(\sum_{i=1}^{n} H \min (i)\right)\right)$

Where $\quad H=$ Height

Hmin=Height of points of low in the neighbourhood of a peak point.

By applying this algorithm, here comes a spatial shape like a pork-pie hat.

A threshold is needed to specify the plane which is considered as the removal border of its floor edges. These edges are problematic in the procedure of locating the second surface momentum in respective of first order point.

In this work by using normal distribution as a model for normal shape of peak and its neighbourhood threshold plane, which is perpendicular to $\mathrm{Z}$ axis of local coordinate system is located in where, the volume between threshold plane and upper surface is the $67 \%$ of its total volume. This is the amount of volume between the $\pm \sigma$. After exertion of these reductions on the elevation surface, it results in a typical shape like below (Fig3). Figures 3-a and 3-c display first order of surface momentum point of resulted surface in a cross section of a plane perpendicular to the X-Y plane. The first surface momentum of a normal wave and an inclined one resulted of the impact of surface winds are shown in the figures 3-b and 3-d respectively. 
International Archives of the Photogrammetry, Remote Sensing and Spatial Information Sciences, Volume XXXIX-B8, 2012 XXII ISPRS Congress, 25 August - 01 September 2012, Melbourne, Australia

The second row figures show a similar cross section of surface but indicate the relative location of both first and second order of momentums to be used in direction finding procedure. It is obvious that both orders points in the normal shape are coincident while there is a displacement of both of them in the inclined and affected state. The vector connecting first to second order point introduces the inclination direction of wave's peak in a 3 dimensional space. Portraying this vector on the $\mathrm{X}-\mathrm{Y}$ plane gives the direction of the current in the local coordinate system, which will be transferred in the specific coordinate system for further use. Here are the equations to compute the first and second order surface momentum having the coordinates of points Eq3.

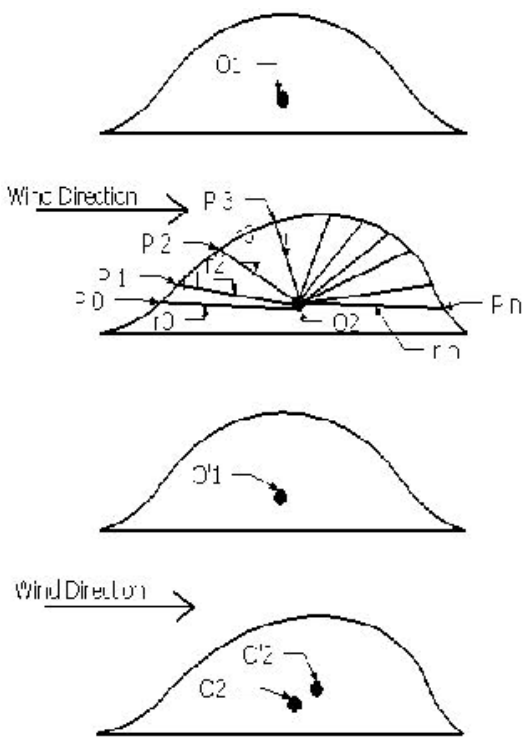

Figure 3. a) First order of surface momentum point of resulted surface of a normal wave in a cross section of a plane perpendicular to the X-Y plane (Most top). b) Distance vector between surface points and First order of surface momentum point of resulted surface of a deformed wave in a cross section of a plane perpendicular to the X-Y plane (Top). c) Second order of surface momentum point of resulted surface of a normal wave in a cross section of a plane perpendicular to the $\mathrm{X}-\mathrm{Y}$ plane (Lower). d) First and Second order of surface momentum point of resulted surface of a deformed wave in a cross section of a plane perpendicular to the X-Y plane (bottom).

$$
\begin{aligned}
& r(i)=|\underline{\underline{O(k)} \underline{F(i)}}| \\
& \underline{O(k)}=1 / r\left(\sum_{i=1}^{i} \underline{P(i))}\right. \\
& \underline{O^{\prime}(k)}-1 / n\left(\sum_{i=1}^{n} r(i) \times \underline{P(i))}\right.
\end{aligned}
$$

Where r(i) Distance between the first momentum and surface Points

$\mathrm{O}(\mathrm{k}) \quad$ First surface momentum point of kth maximum point

$\mathrm{O}^{\prime}(\mathrm{k})$ Second surface momentum point of kth maximum point
P(i) The ith point of surface peak

The second order surface momentum inclines toward the sharpness tip of a deformed shape. Finally to get a better and more reliable result out of this method, the surface model can be accompanied with the bathymetric models of the area.

\section{THE DATA USED AND RESULTS}

The method described above must be applied on a dataset in order to see its impact and results in a real case. For some reasons the TerraSAR-X dataset was not accessible and we were obliged to use another satellite data. The data used in this paper is from Jason1 satellite altimeter and was taken from the NOAA [14] website. Hope to use the TerraSAR-X data in the future works. The Surface model is determined using the Delaunay triangulation method. This surface has been used in our method and the results which were in the local datum have been transferred in to the UTM system. For better demonstration and use of dataset results, the vector field has been exported in the KML format to be shown in the Google Earth Environment. The location of data set is over the Hormuz strait connecting The Persian Gulf and The Oman Sea. Vector field over the Hormuz Strait is shown in the Figures (4, 5 and 6).

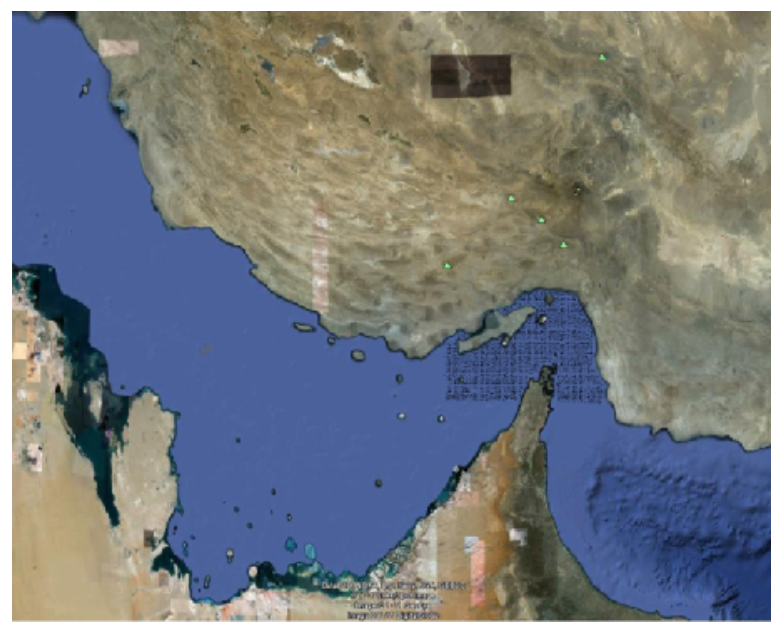

Figure 4. Surface current vector field Over the Coastal area of Hormuz Strait in an overall view and low resolution but high density.

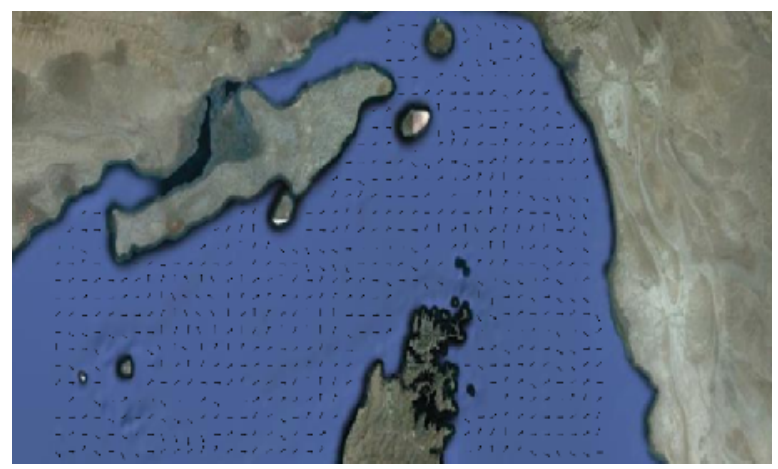

Figure 5. Surface current vector field Over the Coastal area of Hormuz Strait in detailed view and high resolution and vector density. 


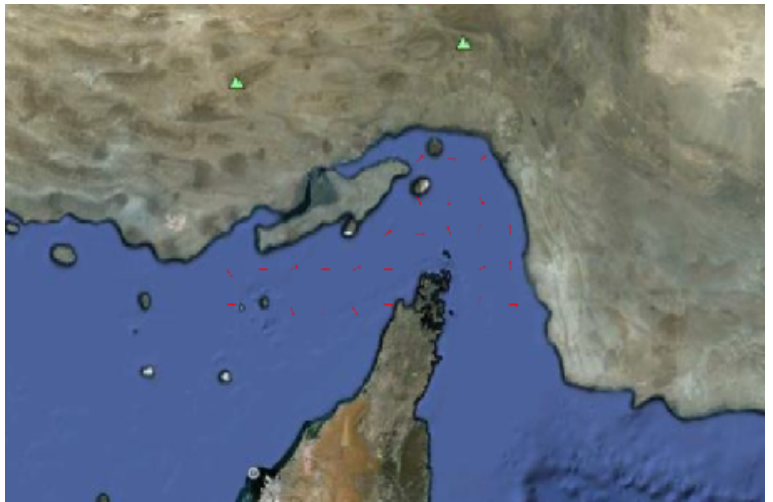

Figure 6. Surface current vector field Over the Coastal area of Hormuz Strait in an overall view and low resolution and vector density.

The analysis limit is set to 55-56 degrees of longitude and a 2627 degree of latitude. The density of vectors in vector field depends on the minimum of planimetry accuracy by satellite and the gridding scale. In figures 4 and 5 vectors are dense and every component of the grid is a $3^{\circ} \times 3^{\circ}$ square while in fig 6 every 25 pixels are averaged in to a pixel.in this figure the scale of each component is $15^{\circ} \times 15^{\circ}$ and vector colour is red.

The output is promising as it has accordance with dominant winds direction of the area.

\section{FUTURE WORKS}

In the future works it would be a suggestion to work on a method to find out the currents velocity out of surface models through geometrical properties of wave shape. Also to develop an algorithm of limiting the wave's analysis range to have more accurate and time saving results in the computations. There is even a potential of study on the higher order momentums and their impact on the results ${ }^{[15][16]}$.

\section{CONCLUSION}

There have been various approaches of determining parameters related to ocean's surface waters. Meanwhile some of them are concentrated on the currents velocity and direction using Doppler Effect or the Satellite Image Based Feature Tracking methods.

The method presented in this paper unlike the Doppler Effect method can give results even with one satellite data while the Doppler Effect needs more than one satellite to get a unidirectional result, because every Doppler observation gives the direction and velocity in the radius path with respect of sensor and for a unidirectional result more than a satellite altimeter or sensor is needed.

Accuracy of the Satellite Image Based Feature Tracking method is dependent on the temporal resolution of images taken of an area and doesn't give a result in bad weather conditions. It also needs a bunch of complex and time consuming feature tracking computations which may get in to mistakes and needs human observation whilst this method's results has a more reliable results in comparison to the Satellite Image Based Feature Tracking method.

The Doppler approach achieves sub centimetre parts of surface water velocity and direction which is a high resolution observation while determining the direction of surface waters needs at least a few meters of resolution in the observations. This quality lets the Doppler method have a significant redundancy and over data in the process which is good but unnecessary.

Altimetry satellites have a history and record of almost 18 year which some of them earlier doesn't include Doppler and SAR observations but elevations. By this method it is possible to use these elevations for direction finding in the coastal areas.

\section{REFFERENCESS}

[1] V. Rosmorduc, J. Benveniste, E. Bronner, S. Dinardo, O. Lauret, C. Maheu, M. Milagro, N. Picot., 2011. Radar Altimetry Tutorial. ESA, Europe, pp. 112-128.

[2] V. Rosmorduc, J. Benveniste, E. Bronner, S. Dinardo, O. Lauret, C. Maheu, M. Milagro, N. Picot., 2011. Radar Altimetry Tutorial. ESA, Europe, pp. 50-52.

[3] Collard Fabrice, Ardhuin Fabrice, Chapron Bertrand (2005). Extraction of coastal ocean wave fields from SAR images. IEEE Journal of Oceanic Engineering, 30(3), 526-533.

[4] Breaker,L.C., V.M.Krasnopolsky and E.M.Maturi, GOES-8 Imagery as a New Source of Data to Conduct Ocean Feature Tracking. Accepted for publication in Remote Sensing of Environment, 1999.

[5] Deng, X., and W.E. Featherstone. 2006. A coastal retracking system for satellite radar altimeter waveforms: Application to ERS-2 around Australia. Journal of Geophysical Research 111, C06012, doi:10.1029/2005JC003039.

[6] NOAA. Feb 2009. The California Coastal Ocean Currents Monitoring Program: An Introduction. pp.4-7.

[7] Cheng Penggen, Liu Shaohua, Wang Wei, Chen Honghua. Construction of three-dimensional geological model of the Research and Application [J]: Journal of Jilin University: Earth Sciences, 2004.4.

[8] Fieguth, P.W., Karl, W.C., Willsky, A.S., Wunsch, C., Lab. for Inf. \& Decision Syst., MIT, Cambridge, MA.. Multiresolution optimal interpolation and statistical analysis of TOPEX/POSEIDON satellite altimetry. Geoscience and Remote Sensing, IEEE Transactions on. Mar 1995.

[9] Li Yan. Based on the Triangular Grid Digital Elevation Model of the Terrain Modeling: World academy of science, Engineering and Technology 452008

[10] Yan Li, Lianhe Yang. Based on Delaunay Triangulation DEM of Terrain Model : Canadian Center of Science and Education(CCSE) Journal. 2009.

[11] Richard Aster, Brian Borchers." Analysis of Time Series and Spatial Data (Geophysics 505/Math 587)',. New Mexico Institute of Mining and Technology Socorro, New Mexico 
International Archives of the Photogrammetry, Remote Sensing and Spatial Information Sciences, Volume XXXIX-B8, 2012 XXII ISPRS Congress, 25 August - 01 September 2012, Melbourne, Australia

http://www.ees.nmt.edu/outside/Geop/Classes/GEOP505.html

[12] Simon Ekholm, Jonathan L. Bamber, William B. Krabill.

The use of airborne laser data to calibrate satellite radar

altimetry data over ice sheets. Journal of Geodynamics 34 (2002) 377-390

[13] International Doris Service. Peer-reviewed journals. http://ids-doris.org/report/publications/peer-reviewedjournals.html

[14] National Oceanic and Atmospheric Administration (NOAA) https://www.noaa.gov/

[15] Hussam Khonkar. Complete Survey of Wind Behavior over the Persian Gulf. JKAU: Mar. Sci., Vol. 20, pp: 31-47 (2009 A.D. / 1430 A.H.)

[16] Ports \& Maritime Organization, Monitoring and Modelling, Study of Iranian Coasts,http://irancoasts.pmo.ir/pg3pg32-pg323-model2-forcast-fa.html 